High-energy physics

\section{Mixed beauty at Hamburg}

\author{
David J. Miller
}

Among the many exciting developments in particle physics reported at a recent meeting*, the most interesting came from the host organization, DESY (Deutsches Elektronen-Synchrotron). The ARGUS collaboration there has seen positive evidence of the 'mixing' of a meson, containing a beauty quark, with its antiparticle. ARGUS has also found a surprisingly large rate for the production of baryons containing beauty quarks. The standard model of particle physics survives intact for another year, having passed several new tests with no serious contradiction of its ingredients: electroweak theory, quantum chromodynamics and the number of quark 'generations'. S.L. Glashow (Harvard) suggested three alternative "fables" of what might emerge at the next generation of accelerators.

Particle-antiparticle mixing has been studied for more than 20 years with $\mathrm{K}^{\mathrm{i}}$ mesons. These particles consist of a down

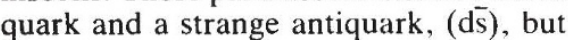
when they decay they sometimes appear to have changed into the $\overline{\mathrm{K}}^{0}$ antiparticle ( $\overline{\mathrm{d} s})$. In their decays they behave like two orthogonal linear combinations of $\mathrm{K}^{0}$ and $\overline{\mathbf{K}}^{0}$, called $\mathrm{K}$-long and $\mathrm{K}$-short because of their different lifetimes. An early success of the standard model was to explain $\mathrm{K}^{0}-\overline{\mathbf{K}}^{0}$ mixing at the same time as predicting the charmed quark. Now the ARGUS group (W. Schmidt-Parzefall, DESY) has seen three distinct independent signals for $\mathrm{B}^{\prime \prime}-\overline{\mathrm{B}}^{\prime \prime}$ mixing $\left(\overline{\mathrm{B}}^{0}\right.$ is a meson with down antiquark and beauty quark ( $\overline{\mathrm{d}} b) ; \mathrm{B}^{\prime \prime}$ is $\left.\mathrm{d} \overline{\mathrm{b}}\right)$.

In both $\mathrm{B}^{0}$ and $\mathrm{K}^{\circ}$ decays, the identity of the decaying quark (particle or antiparticle) is most clearly defined when it decays to a charged lepton (either an electron or a muon), accompanied by a lighter quark and a neutrino (or antineutrino). This is similar to nuclear $\beta$-decay, in which an electron $\left(\mathrm{e}^{-}\right)$and an antineutrino are produced when a down quark in a neutron (udd) turns into an up quark in a proton (uud). Down, strange and beauty' quarks, all being negative, decay to give a negative electron or muon $\left(\mu^{-}\right)$, but their antiquarks give a positive lepton $\left(\mathrm{e}^{+}, \mu^{+}\right)$.

The ARGUS group produced pairs of $\mathrm{B}^{0}$ and $\overline{\mathrm{B}}^{0}$ in $\mathrm{e}^{+}-\mathrm{e}^{-}$collisions at an energy where no extra particles can be produced. In the absence of mixing, one would expect the decay particles to include pairs of leptons of unlike-sign (one from the particle, the other from the antiparticle). But in one sample the group saw 5 likesign lepton pairs and 23 with unlike-sign. The group also achieved a rare success: a

- International Symposium on Lepton and Photon Interactions at High Energy, 27-31 July 1987, Hamburg, FRG fully reconstructed event in which enough tracks could be observed to give a unique identification (see figure). Both decaying particles were $B^{0}$, though one of them must have been produced as $\overline{\mathbf{B}}^{0}$. The UA1 collaboration at CERN (quoted by Schmidt-Parzefall) also reports a like-sign dimuon signal that can be interpreted as $\mathrm{B}^{0}-\overline{\mathrm{B}}^{0}$ mixing, but not as clearly.

These results put tight constraints on some of the arbitrary parameters of the standard model; in particular on the parameters of the unitary matrix (the Cabibbo-Kobayashi-Maskawa matrix)

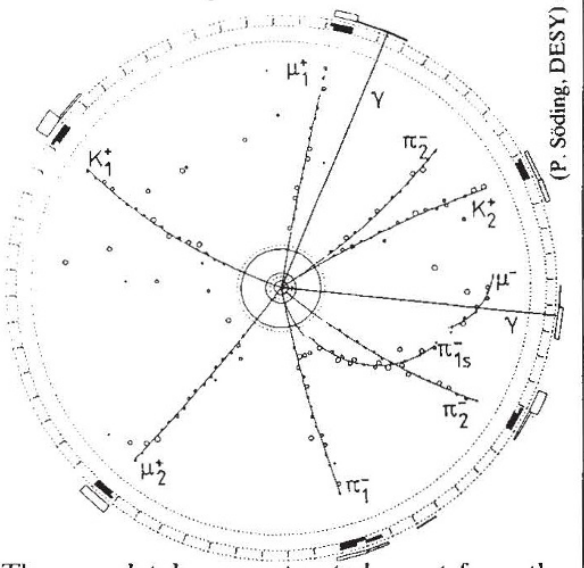

The completely reconstructed event from the ARGUS experiment in which $\mathrm{B}^{0}-\overline{\mathrm{B}}^{0}$ mixing was identified. $\mathrm{A} \mathrm{B}^{0}-\overline{\mathrm{B}}^{0}$ pair is created by an electron-positron collision. The antiparticle turns into a particle by mixing. The two particles then undergo a series of decays to emit the detected particles $\mu_{1}^{+}, \pi_{1}^{-}, \pi_{1}^{-}, \mathrm{K}_{1}^{+}$in the first case and $\mu_{2}^{+}, \pi_{2}^{*}, \pi_{2}, \mathrm{~K}_{2}^{+}$and two photons $\gamma$ in the second. The open circles mark the detected track and the solid lines the computed track.

that describes the weak couplings of all the quarks. The matrix can still be kept unitary (M. Schifman, Institute of Theoretical and Experimental Physics, Moscow; A. Sirlin, New York University), which is necessary if the theory is to conserve the overall sum of quarks and antiquarks, but only if another free parameter of the model is constrained: the mass of the top quark would have to be at least $50 \mathrm{GeV}$, contradicting the CERN claim (now retracted) to have discovered top between 30 and $50 \mathrm{GeV}$ at UA1 (see Nature 311, 210; 1984). This puts the threshold for $t \bar{t}$ production out of range of the Stanford Linear Collider now tuning up, but within range of Large ElectronPositron project (LEP) phase 2 at CERN.

Researchers at CERN have made an improved measurement of the violation of ' $\mathrm{CP}$ ' symmetry, in which the behaviour of a particle is indistinguishable from that of its antiparticle, as seen in a mirror. The spontaneous breaking of this symmetry (first observed in K-meson decays in 1964) may be a clue to the physics of the cosmological Big Bang (reviewed by M.S. Turner, Chicago University). The parameter in the Cabibbo-Kobayashi-Maskawa matrix that describes CP violation should cause a small difference between the decay rates of $\mathrm{K}$-short mesons to two charged or two neutral pions; a difference that was confirmed for the first time in the CERN experiment (I. Mannelli, University of Pisa).

In principle, $\mathrm{CP}$ violation should be observed in B-meson decays, and its rate can be predicted as the mixing parameters are known. Present accelerators, however, would take 100 years to supply the data to measure the effect (SchmidtParzefall) and a new generation of medium-energy, high-luminosity, electron-positron colliders is proposed to complement the high-energy ones being constructed now (described recently in News and Views: Nature 329, 107; 1987).

The newest result reported at the meeting is also the hardest to fit into the standard model (Schifman). The ARGUS collaboration has measured an unexpectedly high rate for the production of pairs of beauty baryon and beauty antibaryon. (Beauty baryons contain a beauty quark and two light quarks; bud, for example.) But there is much work to be done, both experimental and theoretical, before this can be taken seriously. Schifman spent some time explaining how the intrinsic complexity of the "kitchen of strong interactions" destroys some of the simple predictions of pure electroweak theory, and may affect beauty baryon production.

Oddly enough, a similar concept of Schifman's kitchen, but called 'weather' (also denoting nonlinear fluctuations at an unobservably small scale) was introduced by D. Schramm (University of Chicago) to explain disagreements between the standard model of supernovae and the optical observations of the recent supernova 1987A. The neutrino signals are thought to come through the weather like radar through fog, and agree very well with models of the simple primary process. Glashow's three fables, each a minimal alteration of the standard model within present experimental constraints, were presented as examples of the new physics (heavy decaying neutrinos; extra Higgs particles; 'chiral colour' giving a new particle zoo) that could emerge at LEP, or the proposed Superconducting Supercollider in the United States. Although explaining some features of the standard model, the fables avoid any reference to such ambitious (and experimentally unsupported) schemes as technicolour, supersymmetry or superstrings. $\square$

David J. Miller is in the Department of Physics and Astronomy, University College London, Gower Street, London WCIE 6BT, UK. 